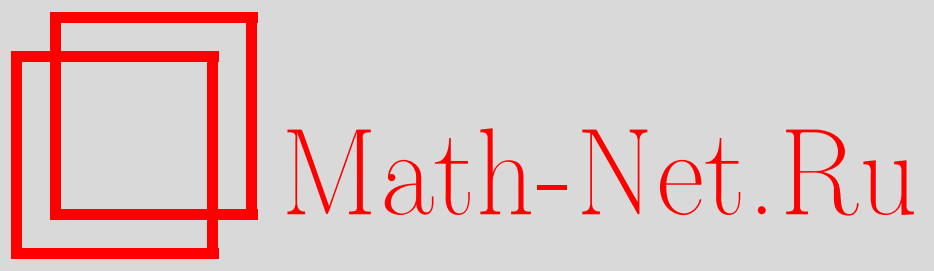

Р. Эрнандес Эредеро, Д. Леви, К. Шимитерна, Классификация дискретных систем на квадратных решетках, TMФ, 2012, том 172, номер 2, 250-263

DOI: https://doi.org/10.4213/tmf6954

Использование Общероссийского математического портала Math-Net.Ru подразумевает, что вы прочитали и согласны с пользовательским соглашением http://www.mathnet.ru/rus/agreement

Параметры загрузки:

IP : 54.224 .187 .69

26 апреля 2023 г., 12:00:48

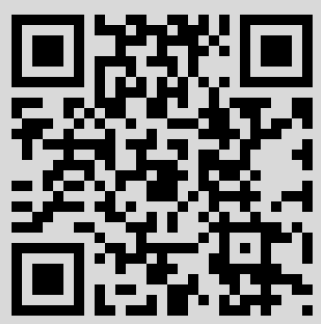


Том 172, № 2

август, 2012

(C) 2012 г. $\quad$ Р. Эрнандес Эредеро*, Д. Леви ${ }^{\dagger}$, К. Шимитерна ${ }^{\dagger}$

\title{
КЛАССИФИКАЦИЯ ДИСКРЕТНЫХ СИСТЕМ НА КВАДРАТНЫХ РЕШЕТКАХ
}

\begin{abstract}
Рассмотрена классификаця с точностью до преобразования Мёбиуса вещественных линеаризуемых и интегрируемых разностных уравнений с дисперсией в частных производных, определенных на квадратной решетке путем мультимасштабной редукции вблизи их гармонического решения. Показано, что условия линеаризуемости и интегрируемости $A_{1}, A_{2}$ и $A_{3}$ ограничивают число параметров, входящих в уравнение. Однако этих условий недостаточно для полной характеристики подкласса мультилинейных уравнений на квадратной решетке.
\end{abstract}

Ключевые слова: мультимасштабное разложение, разностные уравнения, интегрируемые модели, линеаризуемые модели.

\section{1. ВВЕДЕНИЕ}

В 1991 г. Калоджеро [1] ввел понятие $S$ - и $C$-интегрируемых уравнений для характеристики таких нелинейных дифференциальных уравнений в частных производных (ДУЧП), которые решаются путем метода обратной задачи рассеяния и линеаризуются путем замены переменных. С использованием методов мультимасштабной редукции ему удалось показать, что нелинейное уравнение Шредингера (НУШ)

$$
i \partial_{t} u=K_{2}[u]=\partial_{x x} u+\rho_{2}|u|^{2} u, \quad u=u(x, t),
$$

возникает как универсальное уравнение, которому подчиняется эволюция медленно меняющихся пакетов квазимонохроматических волн в слабонелинейной среде с дисперсией. Данная эволюция следует из члена самого низкого порядка в мультимасштабном пертурбативном разложении данного ДУЧП. Необходимые условия $S$-интегрируемости заключаются в том, чтобы $\rho_{2}$ было вещественным. Если, однако, уравнение является линеаризуемым, то $\rho_{2}=0$ является условием того, что уравнение линейно.

* Departamento de Matemática Aplicada, Universidad Politécnica de Madrid, Escuela Universitaria de Ingeniería Técnica de Telecomunicación, Madrid, Spain.

E-mail: rafahh@euitt.upm.es

${ }^{\dagger}$ Dipartimento di Ingegneria Elettronica, Università degli Studi Roma Tre and Sezione INFN, Roma Tre, Rome, Italy. E-mail: levi@roma3.infn.it, scimiterna@fis.uniroma3.it 
Методы мультимасштабной редукции были использованы для нахождения новых $S$-интегрируемых ДУЧП путем изучения высших порядков в разложении и для доказательства интегрируемости новых нелинейных уравнений [2]-[4]. Возможно, наиболее важный пример такого нелинейного ДУЧП - уравнение Дегаспериса-Прочези [5].

Как было показано в работах [6]-[8], введение многих масштабов на решетке редуцирует заданное дискретное уравнение или к локальному нелинейному разностному уравнению в частных производных (РУЧП) при наложении условия медленного изменения, или к ДУЧП, имеющему дело с $\mathbb{C}^{\infty}$-функциями. Здесь мы выбираем второе, поскольку, как было показано в работах [6], [7], только в этом случае сохраняются условия интегрируемости и линеаризуемости дискретного уравнения.

В случае дискретных уравнений было показано [6]-[14], что можно представить эквивалент теоремы Калоджеро-Экхауса [15], которая утверждает, что нелинейное РУЧП с дисперсией не $S$-интегрируемо, если его мультимасштабное разложение по $\mathbb{C}^{\infty}$-функциям не порождает интегрируемости НУШ в низшем порядке. Более того, на примерах было показано, что мультимасштабное разложение на $\mathbb{C}^{\infty}$-функциях для нелинейного $C$-интегрируемого РУЧП порождает линейное ДУЧП [16].

Более того, в работе [17] были представлены некоторые результаты по классификации линеаризуемых РУЧП с использованием мультимасштабной редукции вблизи гармонического решения.

В настоящей работе мы приводим обзор полученных к настоящему времени результатов по классификации линеаризуемых и интегрируемых РУЧП на квадратной решетке вблизи их гармонических решений. Мы демонстрируем необходимые условия для линеаризуемости и интегрируемости класса вещественных разностных уравнений по переменной $u: \mathbb{Z}^{2} \rightarrow \mathbb{R}$ и ее трех ближайших соседей, определенных на квадратной решетке $\mathbb{Z}^{2}$ :

$$
\mathcal{Q}\left(u_{n, m}, u_{n+1, m}, u_{n, m+1}, u_{n+1, m+1} ; \beta_{1}, \beta_{2}, \ldots\right)=0,
$$

где $\beta_{i}$ - вещественные параметры (см. рис. 1). Условия линеаризуемости и интегрируемости получаются путем мультимасштабного пертурбативного разложения, требующего, чтобы уравнение (2) было асимптотически эквивалентно нелинейному уравнению Шредингера, его иерархии и его симметриям или его линеаризованному аналогу.

Будем полагать, что уравнение (2) является линейно-аффинным по каждой переменной, и наша классификация реализуется с точностью до одновременных преобразований Мёбиуса:

$$
u_{n, m} \mapsto \frac{A u_{n, m}+B}{C u_{n, m}+D} .
$$

В разделе 2 кратко обсуждается мультимасштабное разложение для уравнения (2). Демонстрируются основные идеи построения условий интегрируемости, которые обеспечивают то, что заданное уравнение является $S$ - или $C$-интегрируемым уравнением порядка $A_{j}, j=1,2,3$, т. е. асимптотически удовлетворяет $S$ - или $C$-условиям интегрируемости с точностью до третьего, четвертого или пятого порядка по параметру возмущения. В разделе 3 условия интегрируемости и линеаризуемости применяются к уравнению (2), а также приводится последовательность теорем, задающих условия для постоянных $\beta_{i}$, при которых система является $A_{1-}, A_{2^{-}}$или 


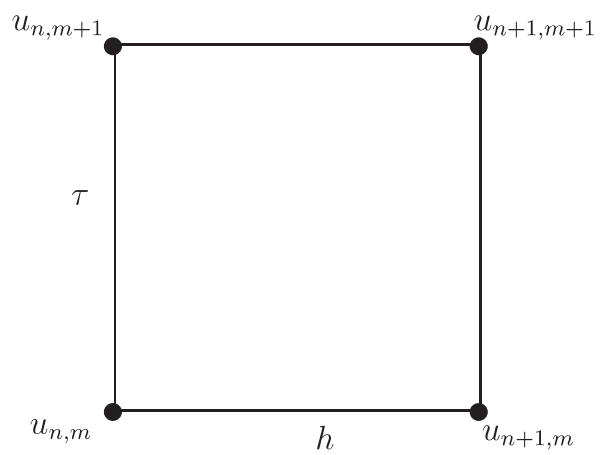

Рис. 1. Квадратная решетка $\mathbb{Z}^{2}$, на которой определено уравнение $\mathcal{Q}=0$.

$A_{3}$-интегрируемой или $A_{1^{-}}, A_{2^{-}}$или $A_{3}$-линеаризуемой. Наконец, в разделе 4 приводятся некоторые заключительные замечания и обсуждаются дальнейшие задачи.

\section{2. РАЗЛОЖЕНИЕ ВЕЩЕСТВЕННЫХ РУЧП}

Теперь кратко проиллюстрируем все составные части метода пертурбативной редукции, необходимые для изучения разностных уравнений, как это представлено в работах [6], [10]. Здесь мы рассмотрим мультимасштабное разложение в случае $\mathbb{C}^{\infty}$-функций с тем, чтобы сохранить линеаризуемость или интегрируемость заданного уравнения.

Вводя множественные масштабы решетки, в рамках некоторых очевидных предположений по поводу $\mathcal{C}^{\infty}$-свойств функции $u_{n, m}$ и радиуса сходимости ее разложения Тейлора для всех $n$ - и $m$ - сдвигов, входящих в разностное уравнение (2), можно записать представление в виде рядов сдвинутых значений $u_{n, m}$ вблизи точки $(n, m)$. Выбирая

$$
n_{i}=\varepsilon_{n_{i}} n, \quad \varepsilon_{n_{i}} \doteq N_{i} \varepsilon^{i}, \quad 1 \leqslant i \leqslant K_{n}, \quad m_{\sigma}=\varepsilon_{m_{\sigma}} m, \quad \varepsilon_{m_{\sigma}} \doteq M_{\sigma} \varepsilon^{\sigma}, \quad 1 \leqslant \sigma \leqslant K_{m},
$$

где различные постоянные $N_{i}, M_{\sigma}$ и $\varepsilon$ являются вещественными числами, причем $K_{n}=1$ и $K_{m}=K$ (в конце концов, $K=+\infty$ ), полные операторы сдвига $T_{n}, T_{m}$ можно переписать в терминах частичных операторов сдвига $\mathcal{T}_{n}, \mathcal{T}_{m}$ как

$$
\begin{aligned}
T_{n} & =\mathcal{T}_{n} \mathcal{T}_{n_{1}}^{\left(\varepsilon_{n_{1}}\right)}=\mathcal{T}_{n} \sum_{j=0}^{+\infty} \varepsilon^{j} \mathcal{A}_{n}^{(j)}, \quad \mathcal{A}_{n}^{(j)} \doteq \frac{N_{1}^{j}}{j !} \partial_{n_{1}}^{j}, \\
T_{m} & =\mathcal{T}_{m} \prod_{j=1}^{K} \mathcal{T}_{m_{j}}^{\left(\varepsilon_{m_{j}}\right)}=\mathcal{T}_{m} \sum_{j=0}^{+\infty} \varepsilon^{j} \mathcal{A}_{m}^{(j)}, \\
T_{n} T_{m} & =\mathcal{T}_{n} \mathcal{T}_{m} \mathcal{T}_{n_{1}}^{\left(\varepsilon_{n_{1}}\right)} \prod_{j=1}^{K} \mathcal{T}_{m_{j}}^{\left(\varepsilon_{m_{j}}\right)}=\mathcal{T}_{n} \mathcal{T}_{m} \sum_{j=0}^{+\infty} \varepsilon^{j} \mathcal{A}_{n, m}^{(j)}
\end{aligned}
$$

и в терминах дифференциальных операторов $\mathcal{A}_{m}^{(j)}, \mathcal{A}_{n, m}^{(j)}$. Эти операторы даются подходящими комбинациями $\left(M_{k}^{j} / j !\right) \partial_{m_{k}}^{j}$ (явные выражения и формулы можно найти в работе [10], подробности см. в работе [18]). Для функции $u_{n, m}=u\left(n, m, n_{1}\right.$, 
$\left.\left\{m_{\sigma}\right\}_{\sigma=1}^{K}, \varepsilon\right)$ можно предположить двойное разложение на гармоники по параметру разложения $\varepsilon$ :

$$
u_{n, m}=\sum_{j=1}^{+\infty} \sum_{\theta=-j}^{j} \varepsilon^{j} u_{j}^{(\theta)}\left(n_{1}, m_{\sigma}, \sigma \geqslant 1\right) e^{i \theta(\kappa h n-\omega \tau m)}
$$

при $u_{j}^{(-\theta)}\left(n_{1}, m_{\sigma}, \sigma \geqslant 1\right)=\bar{u}_{j}^{(\theta)}\left(n_{1}, m_{\sigma}, \sigma \geqslant 1\right)$, чтобы обеспечить вещественность $u_{n, m}$, а $h$ и $\tau$ - постоянные решетки соответственно в направлениях $n$ и $m$. Тогда, подставляя явные выражения (4) для операторов сдвига в терминах производных по медленным переменным, получим, что уравнение (2) окажется бесконечной системой зацепленных ДУЧП. Мы далее требуем, чтобы функции $u_{\gamma}^{(\theta)}$ удовлетворяли асимптотическим условиям $\lim _{n_{1} \rightarrow \pm \infty} u_{j}^{(\theta)}=0$ для всех $j$ и $\theta$, и выбираем индекс $j \geqslant 1$ для того, чтобы любая нелинейная часть уравнения (2) входила как возмущение в мультимасштабное разложение.

Таким образом, мультимасштабное разложение нелинейного РУЧП на $\mathbb{C}^{\infty}$-функции порождает непрерывные ДУЧП. Поэтому проверка на мультимасштабные интегрируемость или линеаризуемость требует, чтобы уравнение было $S$-интегрируемым или $C$-интегрируемым, если его мультимасштабное разложение дает иерархию НУШ или его линеаризованную версию. Это становится очевидным, если рассматривать порядки, следующие за теми, при которых для коэффициента при первой гармонике $u_{1}^{(1)}$ получается НУШ или его линеаризованная версия. В работах [2], [19], [20] содержатся различные подходы к мультимасштабным разложениям высших порядков $S$-интегрируемых нелинейных ДУЧП, а в работе [11] рассматривается линеаризуемый случай.

Сформулируем следующее определение.

ОПРЕДЕЛЕНИЕ 1. Говорят, что нелинейное ДУЧП является $S$-интегрируемым, если у него есть нетривиальная пара Лакса и, следовательно, бесконечное число обобщенных симметрий.

Тогда, следуя работе [2], можно порядок за порядком избавиться от всех секулярных слагаемых в редуцированных уравнениях и, таким образом, в соответствии с работами [3], [21] сформулировать следующие теоремы.

Теорема 1. Нелинейное РУЧП с дисперсией является С-интегрируемым, только если его мультимасштабное разложение имеет вид равномерного асимптотического ряда такого, что:

1) амплитуда $u_{1}^{(1)}$ эволюиионирует по медленным временам $m_{\sigma}, \sigma \geqslant 1$, в соответствии с б-м уравнением линейной иерархии Шредингера,

$$
\partial_{m_{\sigma}} u_{1}^{(1)}=(-i)^{(\sigma-1)} B_{\sigma} \partial_{n_{1}}^{\sigma} u_{1}^{(1)}, \quad B_{\sigma} \doteq-\frac{1}{\sigma !} \frac{d^{\sigma} \omega(\kappa)}{d \kappa^{\sigma}}
$$

где $B_{\sigma}-$ константы; 
2) амплитудъ высших возмущений первых гармоник $u_{j}^{(1)}, j \geqslant 2$, изменяются на медленных временах $m_{\sigma}, \sigma \geqslant 2$, в соответствии с некоторыми линейными неоднородными уравнениями при учете соответствующих асимптотиических граничных условий:

$$
\partial_{m_{\sigma}} u_{j}^{(1)}-(-i)^{(\sigma-1)} B_{\sigma} \partial_{n_{1}}^{\sigma} u_{j}^{(1)} \equiv M_{\sigma} u_{j}^{(1)}=f_{\sigma}(j)
$$

для всех $j, \sigma \geqslant 2$, где $B_{\sigma} \partial_{n_{1}}^{\sigma} u_{1}^{(j)}-\sigma$-й поток в линейной иерархии Шредингера (6). Все остальные $u_{j}^{(\theta)}, \theta \geqslant 2$, выражсаются в терминах дифференииальных мономов no $u_{\rho}^{(1)}, \rho \leqslant j$.

ТЕОРема 2. Нелинейное РУЧП с дисперсией является S-интегрируемым, только если его мультимасштабное разложение имеет вид равномерного асимптотического ряда такого, что:

1) амплитуда $u_{1}^{(1)}$ эволюиионирует по медленным временам $m_{\sigma}, \sigma \geqslant 1$, в соответствии с б-м уравнением нелинейной иерархии Шредингера,

$$
\partial_{m_{\sigma}} u_{1}^{(1)}=K_{\sigma}\left[u_{1}^{(1)}\right]
$$

где $K_{\sigma}\left[u_{1}^{(1)}\right]-\sigma$-й поток в нелинейной иерархии Шредингера;

2) амплитуды высших возмущений первых гармоник $u_{j}^{(1)}, j \geqslant 2$, эволюционируют по медленным временам $m_{\sigma}, \sigma \geqslant 2$, в соответствии с некоторыми нелинейными неоднородными уравнениями с учетом соответствующих асимптотических граничных условий:

$$
M_{\sigma} u_{j}^{(1)}=f_{\sigma}(j), \quad M_{\sigma} \doteq \partial_{t_{\sigma}}-K_{\sigma}^{\prime}\left[u_{1}^{(1)}\right]
$$

для всех $j, \sigma \geqslant 2$. Все осталъные $u_{j}^{(\kappa)}, \kappa \geqslant 2$, выражсаются в терминах дифференциальных мономов по $u_{\rho}^{(1)}, \rho \leqslant j$.

В уравнениях $(7),(9) f_{\sigma}(j)$ - неоднородный нелинейный вынуждающий член; $B_{\sigma}$, $\sigma \geqslant 1$, введенные в уравнении (6), - комплексные постоянные, а $K_{\sigma}^{\prime}[u] v$ - производная Фрешэ нелинейного члена $K_{\sigma}[u]$ вдоль направления $v$, определяемая как $K_{\sigma}^{\prime}[u] v \doteq d K_{\sigma}[u+s v] /\left.d s\right|_{s=0}$, т. е. линеаризация $K_{\sigma}[u]$ вблизи $u$ по направлению $v$. Уравнения (6), (8) представляют собой иерархию совместных эволюций для функции $u_{1}^{(1)}$. Очевидно, что операторы $M_{\sigma}$, определяемые в уравнениях $(7),(9)$, коммутируют между собой. Таким образом, коль скоро мы зафиксировали индекс $j \geqslant 2$ в системе уравнений $(7),(9)$, из их совместности следуют условия

$$
M_{\sigma} f_{\sigma^{\prime}}(j)=M_{\sigma^{\prime}} f_{\sigma}(j) \quad \forall \sigma, \sigma^{\prime} \geqslant 2,
$$

где, поскольку $f_{\sigma}(j)$ и $f_{\sigma^{\prime}}(j)$ являются функциями различных $u_{j}^{(1)}$ - возмущений коэффициентов фундаментальных гармоник до порядка $j-1$, временнь́е производные $\partial_{t_{\sigma}}, \partial_{t_{\sigma^{\prime}}}$ этих коэффициентов, возникающие соответственно в $M_{\sigma}$ и $M_{\sigma^{\prime}}$, следует исключить, используя эволюционные уравнения (6)-(9) вплоть до $j-1$. Условия коммутативности (10) оказываются проверкой на интегрируемость. Явный вид данных условий можно найти в работах [17], [22]. 


\section{3. АФФИННО-ЛИНЕЙНЫЕ УРАВНЕНИЯ С ДИСПЕРСИЕЙ НА КВАДРАТНОЙ РЕШЕТКЕ}

Рассмотрим вещественное однородное аффинно-линейное уравнение с дисперсией, определенное на квадратной решетке и принадлежащее классу (2). Наиболее общее мультилинейное уравнение класса (2) имеет нелинейность не более чем четвертого порядка. Внесем в его линейную часть решение $u_{n, m}=K^{n} \Omega^{m}$, где $K=e^{i \kappa}$ и $\Omega=e^{-i \omega(\kappa)}$. Получим, что это уравнение является уравнением с дисперсией, если оно однородно и записывается как

$$
\begin{aligned}
\mathcal{Q}_{ \pm}= & a_{1}\left(u_{n, m} \pm u_{n+1, m+1}\right)+a_{2}\left(u_{n+1, m} \pm u_{n, m+1}\right)+ \\
& +\left(\alpha_{1}-\alpha_{2}\right) u_{n, m} u_{n+1, m}+\left(\alpha_{1}+\alpha_{2}\right) u_{n, m+1} u_{n+1, m+1}+ \\
& +\left(\beta_{1}-\beta_{2}\right) u_{n, m} u_{n, m+1}+\left(\beta_{1}+\beta_{2}\right) u_{n+1, m} u_{n+1, m+1}+ \\
& +\gamma_{1} u_{n, m} u_{n+1, m+1}+\gamma_{2} u_{n+1, m} u_{n, m+1}+ \\
& +\left(\xi_{1}-\xi_{3}\right) u_{n, m} u_{n+1, m} u_{n, m+1}+\left(\xi_{1}+\xi_{3}\right) u_{n, m} u_{n+1, m} u_{n+1, m+1}+ \\
& +\left(\xi_{2}-\xi_{4}\right) u_{n+1, m} u_{n, m+1} u_{n+1, m+1}+\left(\xi_{2}+\xi_{4}\right) u_{n, m} u_{n, m+1} u_{n+1, m+1}+ \\
& +\zeta u_{n, m} u_{n+1, m} u_{n, m+1} u_{n+1, m+1}=0,
\end{aligned}
$$

где $a_{1}, a_{2} \in \mathbb{R} \backslash\{0\},\left|a_{1}\right| \neq\left|a_{2}\right|$, - коэффициенты, появляющиеся в линейной части, а $\alpha_{1}, \alpha_{2}, \beta_{1}, \beta_{2}, \gamma_{1}, \gamma_{2}, \xi_{1}, \ldots, \xi_{4}, \zeta$ - одиннадцать вещественных параметров, подлежащих определению с использованием мультимасштабной процедуры, описанной в разделе 2. Линейное дисперсионное соотношение имеет вид

$$
\omega(\kappa)=\operatorname{arctg}\left[\frac{\left(a_{1}^{2}-a_{2}^{2}\right) \sin \kappa}{\left(a_{1}^{2}+a_{2}^{2}\right) \cos \kappa+2 a_{1} a_{2}}\right] .
$$

Будем искать преобразования, оставляющие класс уравнений $\mathcal{Q}_{ \pm}$инвариантным. Они дадут условия эквивалентности для нашей классификации. Хорошо известно, что полиномиальные уравнения инвариантны относительно одновременных преобразований Мёбиуса $u_{n, m} \mapsto\left(A u_{n, m}+B\right) /\left(C u_{n, m}+D\right)$. Однако наш класс уравнений является однородным с ограничением на коэффициенты линейной части. После преобразования Мёбиуса появляется постоянное слагаемое

$$
a_{0}=B^{4} \zeta+2 B^{3} D\left(\xi_{1}+\xi_{2}\right)+B^{2} D^{2}\left[\gamma_{1}+\gamma_{2}+2\left(\alpha_{1}+\beta_{1}\right)\right]+2 B D^{3}\left(a_{1}+a_{2}\right),
$$

и, таким образом, если мы не хотим ограничивать коэффициенты в уравнении, нужно положить $B=0$, чтобы получить $a_{0}=0$. После преобразования Мёбиуса при $B=0$ коэффициенты в $\mathcal{Q}_{ \pm}$принимают вид

$$
\begin{aligned}
a_{1} \mapsto D^{3} a_{1}, \quad a_{2} & \mapsto D^{3} a_{2}, \quad \alpha_{1} \mapsto D^{2}\left[\alpha_{1}+C\left(a_{1}+a_{2}\right)\right], \quad \alpha_{2} \mapsto D^{2} \alpha_{2}, \\
\beta_{1} & \mapsto D^{2}\left[\beta_{1}+C\left(a_{1}+a_{2}\right)\right], \quad \beta_{2} \mapsto D^{2} \beta_{2}, \\
\gamma_{1} & \mapsto D^{2}\left(\gamma_{1}+2 C a_{1}\right), \quad \gamma_{2} \mapsto D^{2}\left(\gamma_{2}+2 C a_{2}\right), \\
\xi_{1} \mapsto D \xi_{1}+ & \frac{1}{2} C D\left[3 C\left(a_{1}+a_{2}\right)+\gamma_{1}+\gamma_{2}+2\left(\alpha_{1}-\alpha_{2}+\beta_{1}\right)\right], \\
\xi_{2} \mapsto D \xi_{2}+ & \frac{1}{2} C D\left[3 C\left(a_{1}+a_{2}\right)+\gamma_{1}+\gamma_{2}+2\left(\alpha_{1}+\alpha_{2}+\beta_{1}\right)\right], \\
\xi_{3} & \mapsto D \xi_{3}+\frac{1}{2} C D\left[C\left(a_{1}-a_{2}\right)+\gamma_{1}-\gamma_{2}+2 \beta_{2}\right],
\end{aligned}
$$




$$
\begin{gathered}
\xi_{4} \mapsto D \xi_{4}+\frac{1}{2} C D\left[C\left(a_{1}-a_{2}\right)+\gamma_{1}-\gamma_{2}-2 \beta_{2}\right], \\
\zeta \mapsto \zeta+C^{2}\left[2 C\left(a_{1}+a_{2}\right)+\gamma_{1}+\gamma_{2}+2\left(\alpha_{1}+\beta_{1}\right)\right]+2 C\left(\xi_{1}+\xi_{2}\right) .
\end{gathered}
$$

Таким образом, наши преобразования эквивалентности, относительно которых мы собираемся классифицировать уравнение $\mathcal{Q}_{ \pm}$, ограничены преобразованиями Мёбиуса вида

$$
u_{n, m} \mapsto \frac{u_{n, m}}{C u_{n, m}+D} .
$$

В настоящей работе мы ограничимся рассмотрением случая $\mathcal{Q}_{+} \cdot$ Случай $\mathcal{Q}_{-}$ будет рассмотрен в последующих работах.

\section{1. Линеаризуемые аффинно-линейные уравнения с дисперсией на} квадратной решетке в классе $\mathcal{Q}_{+}$. Требуя, чтобы в низшем порядке в мультимасштабном разложении получалось линейное уравнение Шредингера, мы приходим к следующей теореме.

Теорема 3. В низшем порядке $\varepsilon^{3}$ необходимые условия для линеаризуемости уравнений $\mathcal{Q}_{+}$дают шесть различных классов уравнений, характеризуемых различными диапазонами значений коэффициентов уравнения $\mathcal{Q}_{+}$. Они имеют следующий вид:

- случай 1:

$$
\begin{aligned}
& \alpha_{2}=\beta_{2}=0, \quad \alpha_{1}=\beta_{1}, \quad \gamma_{1}+\gamma_{2}=2 \beta_{1} \\
& \xi_{1}=\xi_{2}=\frac{\left(a_{1}+a_{2}\right)^{2} \gamma_{1} \gamma_{2}+\left(3 a_{1}-2 a_{2}\right) a_{2} \gamma_{1} \beta_{1}-a_{1}\left(2 a_{1}-3 a_{2}\right) \gamma_{2} \beta_{1}}{4 a_{1} a_{2}\left(a_{1}+a_{2}\right)} \\
& \xi_{3}=\xi_{4}=\frac{-\left(a_{1}-a_{2}\right)\left(a_{1}+a_{2}\right)^{2} \gamma_{1} \gamma_{2}-a_{2}\left(-a_{1}^{2}-5 a_{2} a_{1}+2 a_{2}^{2}\right) \gamma_{1} \beta_{1}+a_{1}\left(2 a_{1}^{2}-5 a_{2} a_{1}-a_{2}^{2}\right) \gamma_{2} \beta_{1}}{4 a_{1} a_{2}\left(a_{1}+a_{2}\right)^{2}} ;
\end{aligned}
$$

- случай 2:

$$
\begin{aligned}
& \alpha_{2}=\beta_{2}=0, \quad \alpha_{1}=\beta_{1}, \quad\left(3 a_{1}-2 a_{2}\right) a_{2}^{2} \gamma_{1}+a_{1}^{2}\left(2 a_{1}-3 a_{2}\right) \gamma_{2}=4 a_{1}\left(a_{1}-a_{2}\right) a_{2} \beta_{1}, \\
& \xi_{1}=\xi_{2}=\frac{\left(a_{1}+a_{2}\right)\left(a_{2}^{2} \gamma_{1}^{2}-a_{1}^{2} \gamma_{2}^{2}\right)+2 a_{2}\left(a_{1}^{2}-2 a_{2}^{2}\right) \gamma_{1} \beta_{1}+2 a_{1}\left(2 a_{1}^{2}-a_{2}^{2}\right) \gamma_{2} \beta_{1}-6 a_{1}\left(a_{1}-a_{2}\right) a_{2} \beta_{1}^{2}}{4 a_{1} a_{2}\left(a_{1}^{2}-a_{2}^{2}\right)} \\
& \xi_{3}=\xi_{4}=\frac{2 a_{1} a_{2}\left(a_{1}+a_{2}\right)\left(\gamma_{1}-\gamma_{2}\right) \beta_{1}+\left(a_{1}-a_{2}\right)\left(a_{2} \gamma_{1}-a_{1} \gamma_{2}\right)^{2}+2 a_{1} a_{2}\left(a_{2}-a_{1}\right) \beta_{1}^{2}}{4 a_{1} a_{2}\left(a_{1}+a_{2}\right)^{2}}
\end{aligned}
$$

- случай 3 :

$$
\begin{aligned}
& \alpha_{2}=\beta_{2}=0, \quad a_{1}=2 a_{2}, \quad \gamma_{1}=\frac{2}{3}\left(\alpha_{1}+\beta_{1}\right), \quad \gamma_{2}=\frac{1}{3}\left(\alpha_{1}+\beta_{1}\right), \\
& \xi_{1}=\xi_{2}=-\frac{-5 \alpha_{1} \beta_{1}+\alpha_{1}^{2}+\beta_{1}^{2}}{6 a_{2}}, \quad \xi_{3}=\xi_{4}=-\frac{\left(\alpha_{1}-2 \beta_{1}\right)\left(2 \alpha_{1}-\beta_{1}\right)}{18 a_{2}} ;
\end{aligned}
$$

- случай 4:

$$
\begin{aligned}
& \alpha_{2}=\beta_{2}=0, \quad 2 a_{1}=a_{2}, \quad \gamma_{1}=\frac{1}{3}\left(\alpha_{1}+\beta_{1}\right), \quad \gamma_{2}=\frac{2}{3}\left(\alpha_{1}+\beta_{1}\right), \\
& \xi_{1}=\xi_{2}=-\frac{-5 \alpha_{1} \beta_{1}+\alpha_{1}^{2}+\beta_{1}^{2}}{6 a_{1}}, \quad \xi_{3}=\xi_{4}=\frac{\left(\alpha_{1}-2 \beta_{1}\right)\left(2 \alpha_{1}-\beta_{1}\right)}{18 a_{1}} ;
\end{aligned}
$$


- случай 5:

$$
\begin{aligned}
& \alpha_{2}=\beta_{2}, \quad \alpha_{1}=\beta_{1}, \quad 2 a_{1}=a_{2}, \quad \gamma_{1}=\frac{2 \beta_{1}}{3}, \quad \gamma_{2}=\frac{4 \beta_{1}}{3}, \\
& \xi_{1}=\frac{3 \beta_{1}^{2}-2 \beta_{2} \beta_{1}+\beta_{2}^{2}}{6 a_{1}}, \quad \xi_{2}=\frac{3 \beta_{1}^{2}+2 \beta_{2} \beta_{1}+\beta_{2}^{2}}{6 a_{1}}, \\
& \xi_{3}=-\frac{\beta_{1}^{2}-6 \beta_{2} \beta_{1}+7 \beta_{2}^{2}}{18 a_{1}}, \quad \xi_{4}=-\frac{\beta_{1}^{2}+6 \beta_{2} \beta_{1}+7 \beta_{2}^{2}}{18 a_{1}}
\end{aligned}
$$

- случай 6:

$$
\begin{aligned}
& \alpha_{2}=-\beta_{2}, \quad \alpha_{1}=\beta_{1}, \quad a_{1}=2 a_{2}, \quad \gamma_{1}=\frac{4 \beta_{1}}{3}, \quad \gamma_{2}=\frac{2 \beta_{1}}{3}, \\
& \xi_{1}=\frac{3 \beta_{1}^{2}+2 \beta_{2} \beta_{1}+\beta_{2}^{2}}{6 a_{2}}, \quad \xi_{2}=\frac{3 \beta_{1}^{2}-2 \beta_{2} \beta_{1}+\beta_{2}^{2}}{6 a_{2}}, \\
& \xi_{3}=\frac{\beta_{1}^{2}+6 \beta_{2} \beta_{1}+7 \beta_{2}^{2}}{18 a_{2}}, \quad \xi_{4}=\frac{\beta_{1}^{2}-6 \beta_{2} \beta_{1}+7 \beta_{2}^{2}}{18 a_{2}} .
\end{aligned}
$$

Шесть соответствующих подклассов уравнений инвариантны относителъно ограниченных преобразований Мёбиуса (22).

Случаи 1-6 являются также $A_{2} C$-интегрируемыми, поскольку в порядке $\varepsilon^{4}$ нет условия линеаризуемости.

В качестве следствия теоремы 3 можно сформулировать следующее очевидное, но важное утверждение.

СлеДСтвиЕ 1. Если коэффициенты $a_{1}, a_{2}, \alpha_{1}, \alpha_{2}, \beta_{1}, \beta_{2}, \gamma_{1}, \gamma_{2}, \xi_{1}, \ldots, \xi_{4}$ уравнений $\mathcal{Q}_{+}$не удовлетворяют одному из условий, заданных уравнениями (23)-(28), то $\mathcal{Q}_{+}$не линеаризуемо.

Заметим, что тривиальное условие линеаризуемости $\alpha_{1}=\alpha_{2}=\beta_{1}=\beta_{2}=\gamma_{1}=$ $\gamma_{2}=\xi_{1}=\xi_{2}=\xi_{3}=\xi_{4}=0$ содержится в уравнениях (23)-(28).

Особенно интересно рассмотреть уравнение с не более чем квадратичной нелинейностью. В этом случае мы получаем следующую теорему.

Теорема 4. Если $\xi_{1}=\xi_{2}=\xi_{3}=\xi_{4}=0$ в уравнениях $\mathcal{Q}_{+}$, то низиими условиями линеаризуемости являются:

- случаи 1а и 1б: $\alpha_{2}=\beta_{2}=0, \alpha_{1}=\beta_{1}, a_{1}=(1 / 4)(7 \pm \sqrt{33}) a_{2}, \gamma_{1}=(1 \mp$ $\sqrt{3 / 11}) \beta_{1}, \gamma_{2}=(1 \pm \sqrt{3 / 11}) \beta_{1}$;

- случаи 2а и 2б: $\alpha_{2}=\beta_{2}=0, \alpha_{1}=\beta_{1}, a_{1}=(3 / 4+1 / \sqrt{2} \pm(1 / 2) \sqrt{1 / 4+3 \sqrt{2}}) a_{2}$, $\gamma_{1}=\mp 2 \sqrt{(1 / 41)(19+18 \sqrt{2})} \beta_{1}, \gamma_{2}= \pm 2 \sqrt{(1 / 41)(19+18 \sqrt{2})} \beta_{1}$.

Случаи 1а и $1 б$ содержатся в случае 1 , а случаи 2 а и $2 б-$ в случае 2.

Теперь рассмотрим члены разложения $\varepsilon^{5}$ для случаев 1-6. Имеем следующую теорему. 
Теорема 5. Наиболее общее $A_{3} C$-интегрируемое уравнение представляется как

$$
\begin{gathered}
\alpha_{1}=\beta_{1}=\frac{\left(a_{1}+a_{2}\right) \gamma_{1}}{2 a_{1}}, \quad \alpha_{2}=\beta_{2}=0, \quad \gamma_{2}=\frac{a_{2} \gamma_{1}}{a_{1}}, \\
\xi_{1}=\xi_{2}=\frac{3\left(a_{1}+a_{2}\right) \gamma_{1}^{2}}{8 a_{1}^{2}}, \quad \xi_{3}=\xi_{4}=\frac{\left(a_{1}-a_{2}\right) \gamma_{1}^{2}}{8 a_{1}^{2}} .
\end{gathered}
$$

Случай (29) представляет собой пересечение случаев (23) и (24). Как следствие результата $(29)$ мы получаем, что уравнение вида $\mathcal{Q}_{+}$, которое удовлетворяет теореме 4 (т. е. дисперсионное уравнение с не более чем квадратичной нелинейностью), никогда не будет $C$-интегрируемым. Путем ограниченного преобразования Мёбиуса

$$
u_{n, m}=\frac{\alpha v_{n, m}+\beta}{\gamma v_{n, m}+\delta}, \quad \alpha=2 a_{1} \delta, \quad \beta=0, \quad \gamma=-\gamma_{1} \delta,
$$

можно получить канонический вид уравнений, относящихся к случаю (29):

$$
v_{n, m}+v_{n+1, m+1}+\epsilon\left(v_{n+1, m}+v_{n, m+1}\right)+\zeta^{\prime} v_{n, m} v_{n+1, m} v_{n, m+1} v_{n+1, m+1}=0,
$$

где $\epsilon \doteq a_{2} / a_{1} \neq 0, \pm 1$ и $\zeta^{\prime} \doteq-2\left[\left(a_{1}+a_{2}\right) \gamma_{1}^{3}-4 a_{1}^{3} \zeta\right] / a_{1}$.

Нетрудно доказать следующую теорему.

Теорема 6. Уравнения $\mathcal{Q}_{+}$, удовлетворяющие $A_{3} C$-интегрируемости $(29)$, можно линеаризовать с помощью вещественного преобразования Мёбиуса тогда и только тогда, когда $\zeta=\left(a_{1}+a_{2}\right) \gamma_{1}^{3} / 4 a_{1}^{3}$, что соответствует $\zeta^{\prime}=0$ в (31). Линеаризующее преобразование задается выражением (30), а результирующее линеаризованное уравнение имеет вид

$$
v_{n, m}+v_{n+1, m+1}+\epsilon\left(v_{n+1, m}+v_{n, m+1}\right)=0 .
$$

Уравнение (32) является наиболее общим линейным уравнением с дисперсией, определенным на квадрате.

\section{2. Интегрируемые аффинно-линейные уравнения с дисперсией на} квадратной решетке в классе $\mathcal{Q}_{+}$. В низшем порядке $\varepsilon^{3}$ условий интегрируемости получаем следующую теорему.

ТЕОРема 7. Необходимые условия в низшем порядке для интегрируемости уравнений $\mathcal{Q}_{+}$имеют следующий вид:

- случай $1(\mathcal{N}=8)$ :

$$
\begin{gathered}
\alpha_{2}=\beta_{2}=0, \\
\xi_{1}=\xi_{2}, \quad \xi_{3}=\xi_{4} ;
\end{gathered}
$$

- случай $2(\mathcal{N}=6)$ :

$$
\begin{gathered}
\alpha_{2}=\beta_{2}, \quad \alpha_{1}=\beta_{1}, \\
a_{1}=2 a_{2}, \\
\gamma_{1}=2 \gamma_{2}, \\
a_{1}\left(\xi_{1}-\xi_{2}\right)=-a_{1}\left(\xi_{3}-\xi_{4}\right)=-2 \alpha_{2} \gamma_{2} ;
\end{gathered}
$$


- случай $3(\mathcal{N}=6)$ :

$$
\begin{gathered}
\alpha_{2}=-\beta_{2}, \quad \alpha_{1}=\beta_{1}, \\
a_{2}=2 a_{1}, \\
\gamma_{2}=2 \gamma_{1}, \\
a_{1}\left(\xi_{1}-\xi_{2}\right)=a_{1}\left(\xi_{3}-\xi_{4}\right)=-\alpha_{2} \gamma_{1} ;
\end{gathered}
$$

- случай $4(\mathcal{N}=7)$ :

$$
\begin{gathered}
a_{2} \alpha_{1}=a_{2} \beta_{1}=\frac{1}{2}\left(a_{1}+a_{2}\right) \gamma_{2}, \\
a_{2} \gamma_{1}=a_{1} \gamma_{2}, \\
a_{1}\left(\xi_{1}-\xi_{2}\right)=-\alpha_{2} \gamma_{1}, \\
a_{1}\left(\xi_{3}-\xi_{4}\right)=\beta_{2} \gamma_{1}
\end{gathered}
$$

- случай $5(\mathcal{N}=7)$ :

$$
\begin{gathered}
\left(a_{2}-a_{1}\right) \beta_{2}=\left(a_{2}+a_{1}\right) \alpha_{2}, \\
2 a_{1} a_{2}\left(a_{1}-a_{2}\right) \alpha_{1}=\left(a_{1}+a_{2}\right)\left(\gamma_{2} a_{1}^{2}-\gamma_{1} a_{2}^{2}\right), \\
2 a_{1} a_{2} \beta_{1}=\gamma_{1} a_{2}^{2}+\gamma_{2} a_{1}^{2}, \\
\left(a_{2}-a_{1}\right)\left(\xi_{1}-\xi_{2}\right)=\left(\gamma_{1}-\gamma_{2}\right) \alpha_{2}, \\
\left(a_{2}-a_{1}\right)^{2}\left(\xi_{3}-\xi_{4}\right)=\left[\gamma_{2}\left(a_{2}-3 a_{1}\right)-\gamma_{1}\left(a_{1}-3 a_{2}\right)\right] \alpha_{2} ;
\end{gathered}
$$

- случай $6(\mathcal{N}=7)$ :

$$
\begin{gathered}
\left(a_{2}+a_{1}\right) \beta_{2}=\left(a_{2}-a_{1}\right) \alpha_{2}, \\
2 a_{1} a_{2} \alpha_{1}=\gamma_{1} a_{2}^{2}+\gamma_{2} a_{1}^{2} \\
2 a_{1} a_{2}\left(a_{1}-a_{2}\right) \beta_{1}=\left(a_{1}+a_{2}\right)\left(\gamma_{2} a_{1}^{2}-\gamma_{1} a_{2}^{2}\right), \\
\left(a_{2}^{2}-a_{1}^{2}\right)\left(\xi_{1}-\xi_{2}\right)=\left[\gamma_{1}\left(a_{1}-3 a_{2}\right)-\gamma_{2}\left(a_{2}-3 a_{1}\right)\right] \alpha_{2}, \\
\left(a_{1}+a_{2}\right)\left(\xi_{3}-\xi_{4}\right)=\left(\gamma_{2}-\gamma_{1}\right) \alpha_{2} .
\end{gathered}
$$

Пусть $\mathcal{N}$ - число свободных параметров, содержащихся в получающемся нелинейном решеточном уравнении. Соответствующие шесть подклассов уравнений инвариантны относительно ограниченных преобразований Мёбиуса (22).

Как и в случае линеаризуемых уравнений, можно сформулировать следующее следствие.

СлеДСтвиЕ 2. Если коэффициенты $a_{1}, a_{2}, \alpha_{1}, \alpha_{2}, \beta_{1}, \beta_{2}, \gamma_{1}, \gamma_{2}, \xi_{1}, \ldots, \xi_{4}$ уравнения $\mathcal{Q}_{+}$не удовлетворяют одному из условий, заданных уравнениями (33)-(35), то $\mathcal{Q}_{+}$не является интегрируемым.

Если $\xi_{1}=\xi_{2}=\xi_{3}=\xi_{4}=0$ в уравнениях $\mathcal{Q}_{+}$, то необходимые условия интегрируемости получающегося уравнения в низшем порядке имеют следующий вид:

- случай 1: $\alpha_{2}=\beta_{2}=0$;

- случай 2: $\alpha_{2}=\beta_{2}, \alpha_{1}=\beta_{1}, a_{1}=2 a_{2}, \gamma_{1}=\gamma_{2}=0$;

- случай 3: $\alpha_{2}=-\beta_{2}, \alpha_{1}=\beta_{1}, 2 a_{1}=a_{2}, \gamma_{1}=\gamma_{2}=0$;

- случай 4: $\alpha_{1}=\beta_{1}, \gamma_{1}=\gamma_{2}=0$. 
Каждый из этих случаев представляет собой в точности то подмножество случаев 1-4 из теоремы 7, которое составлено из квадратных уравнений. Случаи 5 и 6 из теоремы 7 не дают квадратичных подслучаев.

Перейдем к порядку $\varepsilon^{4}$ в пертурбативном разложении. Тогда для классификации $A_{2}$-интегрируемости получим следующий результат.

Теорема 8. Необходимые и достаточные условия для $\varepsilon^{4}$-асимптотики S-интегрируемости имеют следующий вид:

- случай 1:

$$
\alpha_{2}=\beta_{2}=0, \quad \xi_{1}=\xi_{2}, \quad \xi_{3}=\xi_{4} ;
$$

- случай 4:

$$
\begin{gathered}
\alpha_{1}=\beta_{1}=\frac{\left(a_{1}+a_{2}\right) \gamma_{1}}{2 a_{1}}, \quad \gamma_{2}=\frac{a_{2} \gamma_{1}}{a_{1}}, \\
a_{1}\left(\xi_{1}-\xi_{2}\right)=-\alpha_{2} \gamma_{1}, \quad a_{1}\left(\xi_{3}-\xi_{4}\right)=\beta_{2} \gamma_{1} .
\end{gathered}
$$

Таким образом, в порядке $\varepsilon^{4}$ случаи 1 и 4 из теоремы 7 проходят проверку, а случаи $2,3,5$ и 6 переходят в подслучаи 1 и 4 .

В порядке $\varepsilon^{5}$ пертурбативного разложения имеем следующий результат для классификации $A_{3}$-интегрируемости.

Теорема 9. С точностъю до ограниченного преобразования Мёбиуса (22) для $\varepsilon^{5}$ единственнъе асимптотически $S$-интегрируемые случаи даются следующими нормированными уравнениями:

$$
\begin{aligned}
v_{n, m} & +v_{n+1, m+1}+2\left(v_{n+1, m}+v_{n, m+1}\right)+v_{n+1, m} v_{n, m+1}\left(\tau_{1}+\tau_{2}\right)+ \\
& +\left(v_{n+1, m} v_{n+1, m+1}+v_{n, m} v_{n, m+1}\right) \tau_{2}+\left(v_{n, m+1} v_{n+1, m+1}+v_{n, m} v_{n+1, m}\right) \tau_{1}+ \\
& +v_{n+1, m} v_{n, m+1}\left(v_{n, m}+v_{n+1, m+1}\right) \tau_{1} \tau_{2}=0 \\
v_{n, m} & +v_{n+1, m+1}+\frac{1}{2}\left(v_{n+1, m}+v_{n, m+1}\right)+v_{n+1, m} v_{n, m+1}\left(\tau_{1}+\tau_{2}\right)+ \\
& +\left(v_{n+1, m} v_{n+1, m+1}+v_{n, m} v_{n, m+1}\right) \tau_{2}+\left(v_{n, m+1} v_{n+1, m+1}+v_{n, m} v_{n+1, m}\right) \tau_{1}+ \\
& +2 v_{n, m} v_{n+1, m+1}\left(v_{n+1, m}+v_{n, m+1}\right) \tau_{1} \tau_{2}+ \\
& +4 v_{n, m} v_{n+1, m} v_{n, m+1} v_{n+1, m+1}\left(\tau_{1}+\tau_{2}\right) \tau_{1} \tau_{2}=0, \\
v_{n, m} & +v_{n+1, m+1}+\epsilon\left(v_{n+1, m}+v_{n, m+1}\right)+ \\
& +s\left[\epsilon v_{n+1, m} v_{n, m+1}\left(v_{n, m}+v_{n+1, m+1}\right)+v_{n, m} v_{n+1, m+1}\left(v_{n+1, m}+v_{n, m+1}\right)\right]+ \\
& +\zeta^{\prime} v_{n, m} v_{n+1, m} v_{n, m+1} v_{n+1, m+1}=0, \\
v_{n, m} & +v_{n+1, m+1}+\epsilon\left(v_{n+1, m}+v_{n, m+1}\right)+ \\
& +s\left[v_{n+1, m} v_{n, m+1}\left(v_{n, m}+v_{n+1, m+1}\right)+\epsilon v_{n, m} v_{n+1, m+1}\left(v_{n+1, m}+v_{n, m+1}\right)\right]+ \\
& +\zeta^{\prime} v_{n, m} v_{n+1, m} v_{n, m+1} v_{n+1, m+1}=0, \\
v_{n, m} & +v_{n+1, m+1}+\epsilon\left(v_{n+1, m}+v_{n, m+1}\right)+v_{n, m} v_{n+1, m+1}-v_{n+1, m} v_{n, m+1}+ \\
& +\left(1-\frac{1}{\epsilon}\right)\left[v_{n+1, m} v_{n, m+1}\left(v_{n, m}+v_{n+1, m+1}\right)-v_{n, m} v_{n+1, m+1}\left(v_{n+1, m}+v_{n, m+1}\right)\right]+ \\
& +\left(1-\frac{1}{\epsilon^{2}}\right) v_{n, m} v_{n+1, m} v_{n, m+1} v_{n+1, m+1}=0 .
\end{aligned}
$$


В уравнениях (39), (40) $\tau_{1}=0,1 ;$ если $\tau_{1}=0$, то $\tau_{2}=1$. В уравнениях $(41),(42)$ $s=0, \pm 1 u \epsilon \neq 0, \pm 1$. В уравнении $(43) \epsilon \neq 0, \pm 1,2,1 / 2$.

\section{4. ЗАКЛЮЧЕНИЕ}

В настоящей работе представлена полная классификация линеаризуемых и интегрируемых вещественных РУЧП с дисперсией, принадлежащих классу $\mathcal{Q}_{+}$, с использованием мультимасштабных разложений вблизи периодичного дискретного волнового решения линеаризованого уравнения с точностью до пятого порядка по параметру разложения $\varepsilon$.

Вычисления показали, что мультимасштабное разложение можно эффективно использовать для классификации асимптотически линеаризуемых и интегрируемых дискретных уравнений.

Получающаяся $\varepsilon^{5}$-линеаризуемая система зависит от четырех параметров, только два из которых являются существенными, как можно видеть из уравнения (31). Требование, чтобы получающееся уравнение явно линеаризовалось с помощью преобразования Мёбиуса, редуцирует параметры к одному. Доказательство линеаризуемости уравнения $\mathcal{Q}_{+}$, удовлетворяющего в точности условиям $A_{3} C$-интегрируемости (29), остается пока нерешенной задачей. Возможно, переход к более высоким порядкам в пертурбативном разложении позволит нам зафиксировать параметр $\zeta$ согласно теореме 6. Некоторые дополнительные результаты, указываюшие на то, что формулы (29) или (31) не дают линеаризуемых уравнений, приведены в работе [23].

В асимптотически $S$-интегрируемом случае условия $A_{3}$-интегрируемости дают пятимерные инвариантные разностные уравнения Мёбиуса в частных производных с дисперсией, принадлежащие к классу $\mathcal{Q}_{+}$, зависящему от меньшего числа свободных параметров - не более двух. Уравнения (41), (42) при $s=0$ сводятся к (31); более того, при $\zeta^{\prime}=0$ они исследовались в работах [24], [25], где было показано, что они $S$-интегрируемы. Другие случаи, насколько нам известно, являются новыми, и их интегрируемость должна быть доказана другими методами (ведь коль скоро мы не в состоянии добраться до бесконечного порядка, метод мультимасштабного разложения дает лишь необходимые, но не достаточные условия).

Кроме того, ведется работа по исследованию случая $\mathcal{Q}_{-}$. В этом случае мы получаем для низшего порядка по пертурбативному параметру нелинейную систему ДУЧП, связывающую фундаментальную гармонику с нулевой. Решение этого уравнения является ключевой составляющей классификации данного класса уравнений, содержащего все уравнения с дисперсией, принадлежащие $A B S$-классификации мультилинейных уравнений на квадрате [26].

Более фундаментальный интересный класс РУЧП, который следует рассмотреть, - это уравнения, определенные на треугольнике, т. е. уравнения относительно переменной $u: \mathbb{Z}^{2} \rightarrow \mathbb{R}$ и двух ближайших соседей:

$$
\mathcal{Q}\left(u_{n, m}, u_{n+1, m}, u_{n, m+1} ; \beta_{1}, \beta_{2}, \ldots\right)=0
$$

где $\beta_{i}$ - вещественные параметры. Нетрудно видеть, что для них нет гармонического решения для линейной части (44) и, следовательно, в этом случае не имеется и представленного здесь мультимасштабного разложения. В этом случае можно 
использовать другие методы. Классификацию интегрируемых уравнений можно получить, используя формальный симметрийный подход (см. работу [24] для случая на квадрате), а классификацию линеаризуемых уравнений - за счет требования о существовании линеаризующего преобразования (см. работу [23] для случая на квадрате). Мы работаем в этом направлении.

Благодарности. Работа выполнена при частичной финансовой поддержке Italian Ministry of Education и Research, PRIN "Непрерывные и дискретные нелинейные интегрируемые эволюции: от волн на воде до симплектических отображений”, начиная с 2010 г. Д. Леви выражает благодарность за поддержку во время своего пребывания в Мадриде. Р. Эрнандес Эредеро выражает благодарность Д. Леви и INFN, Sezione Roma Tre за поддержку во время своего пребывания в Риме.

\section{Список литературы}

[1] F. Calogero, "Why are certain nonlinear PDEs both widely applicable and integrable?", What is Integrability?, Springer Series in Nonlinear Dynamics, ed. V. E. Zakharov, Springer, Berlin, 1991, 1-62.

[2] A. Degasperis, S. V. Manakov, P. M. Santini, Physica D, 100:1-4 (1997), 187-211.

[3] A. Degasperis, M. Procesi, "Asymptotic integrability", Symmetry and perturbation theory, SPT 98, eds. A. Degasperis, G. Gaeta, World Scientific, Singapore, 1999, 23-37; A. Degasperis, "Multiscale expansion and integrability of dispersive wave equations", Integrability, Lecture Notes in Physics, 767, ed. A. V. Mikhailov, Springer, Berlin, 2009, 215-244.

[4] Y. Kodama, A. V. Mikhailov, "Obstacles to asymptotic integrability", Algebraic Aspects of Integrable Systems, Progress in Nonlinear Differential Equations and their Applications, 26, eds. A. S. Fokas, I. M. Gelfand, Birkhäuser, Boston, MA, 1997, 173-204; Y. Hiraoka, Y. Kodama, "Normal form and solitons", Integrability, Lecture Notes in Physics, 767, ed. A. V. Mikhailov, Springer, Berlin, 2009, 175-214.

[5] А. Дегасперис, Д. Д. Холм, А. Хон, ТМФ, 133:2 (2002), 170-183.

[6] R. Hernández Heredero, D. Levi, M. Petrera, C. Scimiterna, J. Phys. A, 41:31 (2008), 315208, 12 pp.

[7] R. Hernández Heredero, D. Levi, M. Petrera, C. Scimiterna, J. Nonlinear Math. Phys., 15:suppl.3 (2008), 323-333.

[8] D. Levi, J. Phys. A, 38:35 (2005), 7677-7689, arXiv: nlin/0505061.

[9] M. Agrotis, S. Lafortune, P. G. Kevrekidis, Discrete Contin. Dyn. Syst., suppl. (2005), $22-29$.

[10] R. Hernández Heredero, D. Levi, M. Petrera, C. Scimiterna, J. Phys. A, 40:34 (2007), F831-F840.

[11] J. Leon, M. Manna, J. Phys. A, 32:15 (1999), 2845-2869.

[12] D. Levi, R. Hernández Heredero, J. Nonlinear Math. Phys., 12:suppl. 1 (2005), 440-448.

[13] D. Levi, M. Petrera, J. Math. Phys., 47:4 (2006), 043509, 20 pp., arXiv: math-ph/0510084.

[14] S. W. Schoombie, J. Comput. Phys., 101:1 (1992), 55-70.

[15] F. Calogero, W. Eckhaus, Inverse Problems, 3:2 (1987), L27-L32; 3:2 (1987), 229-262; 4:1 (1987), 11-33; F. Calogero, A. Degasperis, X. Ji, J. Math. Phys., 42:6 (2001), 2635-2652; 41:9 (2000), 6399-6443; F. Calogero, A. Maccari, "Equations of nonlinear Schrödinger type in $1+1$ and $2+1$ dimensions obtained from integrable PDEs", Inverse Problems: an Interdisciplinary Study, Proceedings of the meeting (Montpellier, December 1-5, 1986), Advances in Electronics and Electron Physics, suppl. 19, ed. P. C. Sabatier, Academic Press, London, 1987, 463-480.

[16] C. Scimiterna, D. Levi, SIGMA, 6 (2010), 070, 17 pp., arXiv: 1005.5288. 
[17] R. Hernández Heredero, D. Levi, C. Scimiterna, J. Phys. A, 43:50 (2010), 502002, 14 pp., arXiv: 1011.0141.

[18] C. Scimiterna, Multiscale techniques for nonlinear difference equations, $\mathrm{Ph}$. D. thesis, Roma Tre Universitá Degli Studi, Rome (Italy), 2009,

http://www.fis.uniroma3.it/dottorato_tesi/Scimiterna_SCIMITERNA.pdf.

[19] Y. Kodama, Phys. Lett. A, 107:6 (1985), 245-249.

[20] Y. Kodama, "Nearly integrable systems: normal form and solitons", Nonlinear Evolutions, Proceedings of the Fourth Workshop on Nonlinear Evolution Equations and Dynamical Systems (Balaruc-les-Bains, June 11-25, 1987), ed. J. J. P. Leon, World Scientific, Teaneck, NJ, 1988, 559-570.

[21] M. Procesi, Non linear waves, multiscale methods and integrability master degree, Ph.D. thesis, Physics Department, "La Sapienza" University, Rome, 1997.

[22] D. Levi, C. Scimiterna, Appl. An., 89:4 (2010), 507-527.

[23] D. Levi, C. Scimiterna, SIGMA, 7 (2011), 079, 24 pp.

[24] D. Levi, R.I. Yamilov, J. Phys. A, 42:45 (2009), 454012, 18 pp., arXiv: 0902.4421; 44:14 (2011), 145207, 22 pp., arXiv: 1011.0070.

[25] C. Scimiterna, B. Grammaticos, A. Ramani, J. Phys. A, 44:3 (2011), 032002, 6 pp.

[26] V.E. Adler, A. I. Bobenko, Yu. B. Suris, Commun. Math. Phys., 233:3 (2003), 513-543, arXiv: nlin/0202024. 